Electronic Supporting Information

for

\title{
Quantitative Control of Gene-Engineered T cell Activity Through Covalent Attachment of Targeting Ligands to a Universal Immune Receptor
}

Nicholas G. Minutolo ${ }^{1,2,3}$, Prannda Sharma ${ }^{1,2}$, Mathilde Poussin ${ }^{1,2}$, Lauren C. Shaw ${ }^{1,2,3}$, Daniel P. Brown $^{1,2,3}$, Erin E. Hollander ${ }^{4}$, Anže Smole ${ }^{1,2,5}$, Alba Rodriguez-Garcia ${ }^{1,2}$, James Z. Hui ${ }^{6}$, Fabiana Zappala $^{6}$, Andrew Tsourkas ${ }^{6}$, Daniel J. Powell, Jr. ${ }^{1,2,7^{*}}$

${ }^{1}$ Department of Pathology and Laboratory Medicine, Perelman School of Medicine, University of Pennsylvania, Philadelphia, PA 19104, USA

${ }^{2}$ Center for Cellular Immunotherapies, University of Pennsylvania School of Medicine, Philadelphia, PA 19104, USA

${ }^{3}$ Department of Systems Pharmacology and Translational Therapeutics, University of Pennsylvania School of Medicine, Philadelphia PA 19104, USA

${ }^{4}$ Department of Cancer Biology, University of Pennsylvania School of Medicine, Philadelphia, PA, United States

${ }^{5}$ Parker Institute for Cancer Immunotherapy, Perelman School of Medicine, University of Pennsylvania, Philadelphia, PA, 19104, USA

${ }^{6}$ Department of Bioengineering, University of Pennsylvania, Philadelphia, PA 19104, USA

${ }^{7}$ Lead contact

* Correspondence: poda@pennmedicine.upenn.edu

Daniel Powell, $\mathrm{PhD}$

University of Pennsylvania

3400 Civic Center Blvd.

Bldg. 421, Smilow Translational Research Center, Rm 8-103

Philadelphia, PA 19104-5156

Office: $215-573-4783$

Fax: 215-573-5129 


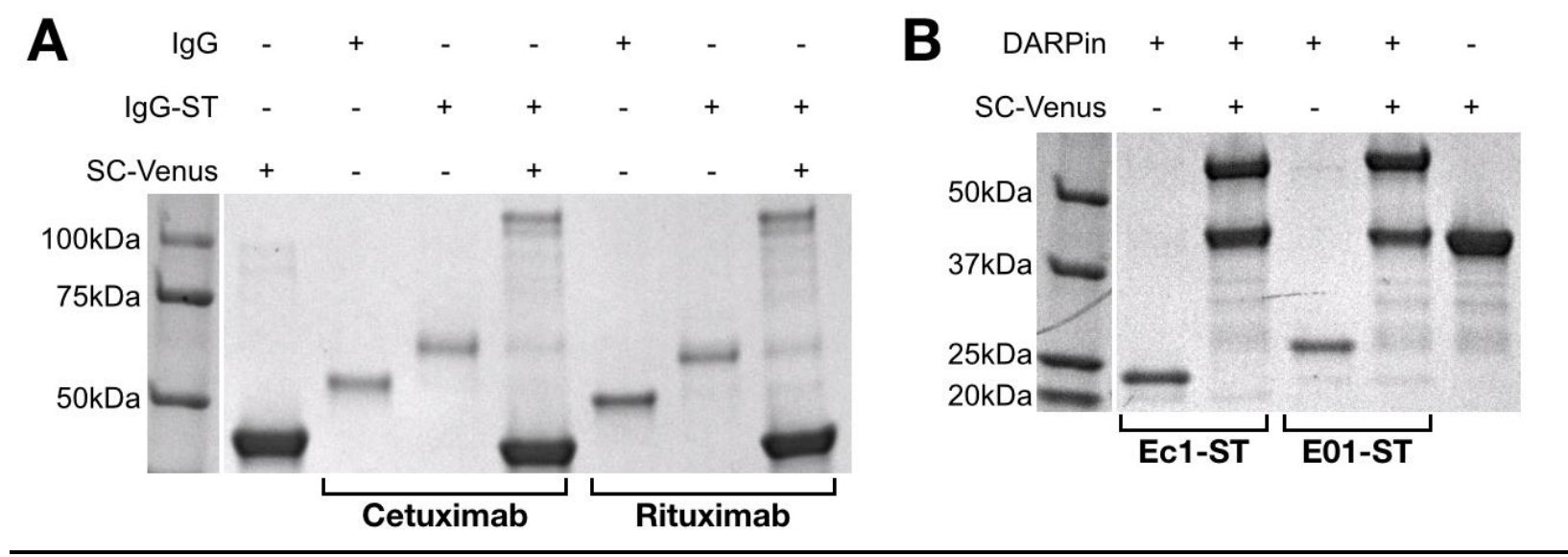

Supplemental Figure 1. Production of SpyTag-labeled targeting ligands. (A) Crosslinking of Rituximab or Cetuximab with Protein G-ST, followed by subsequent reaction with SpyCatcher-Venus analyzed by SDS-Page gel under reducing conditions with Coomassie staining. (B) DARPins Ec1-ST and E01-ST reacted with SpyCatcher-Venus analyzed by SDS-Page gel with Coomassie staining 
A)
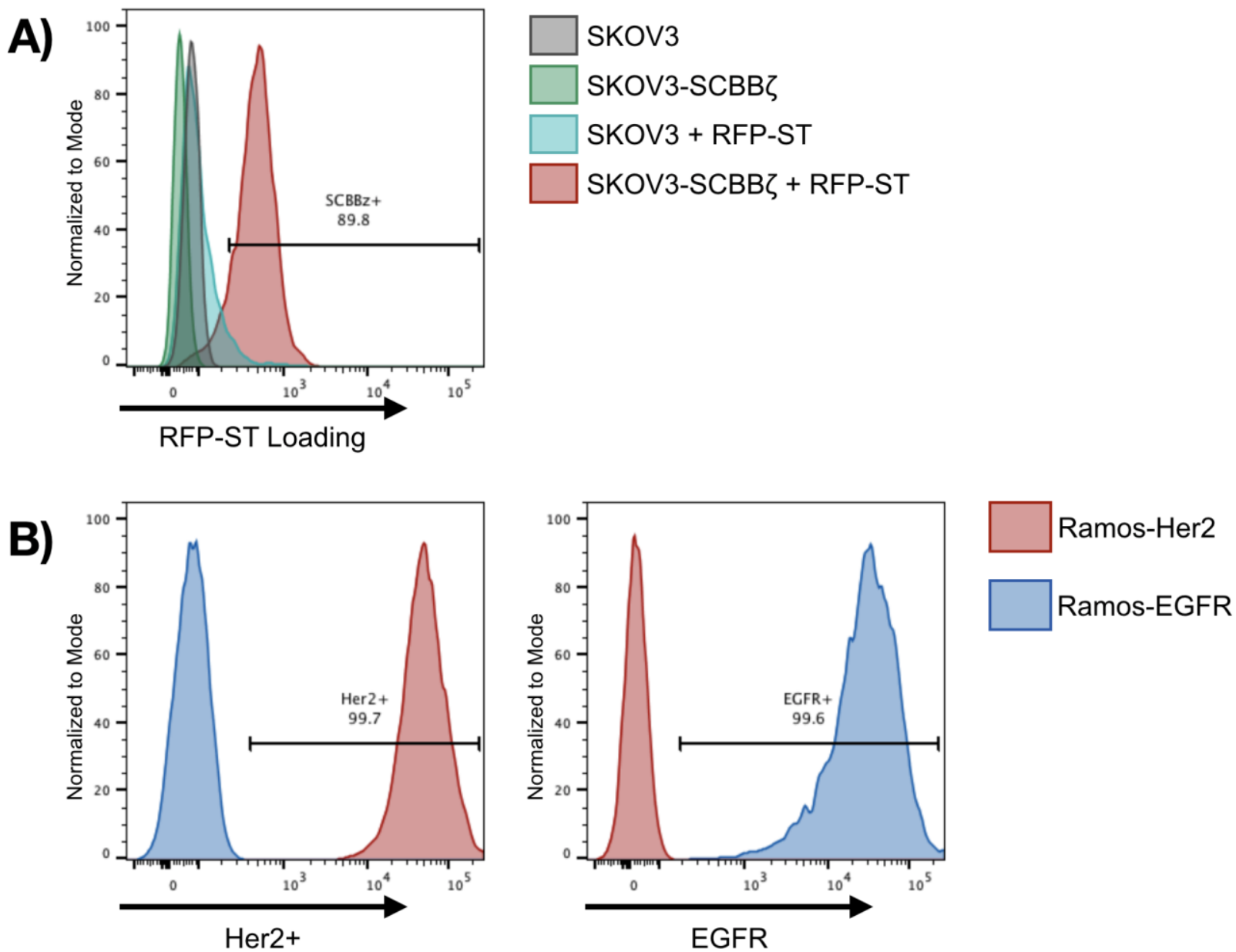

Supplemental Figure 2. Expression of proteins in stable cell lines via lentiviral transduction. (A) SKOV3-GFP and SKOV3-GFP-SpyCatcher-BB $\zeta$ cells were incubated in culture medium containing 2000nM myc-RFP-ST. Cells were stained for receptor arming via anti-myc antibody and analyzed by flow cytometry. (B) Ramos-Her2 and Ramos-EGFR cells co-stained with anti-Her2 and anti-EGFR monoclonal antibodies. 

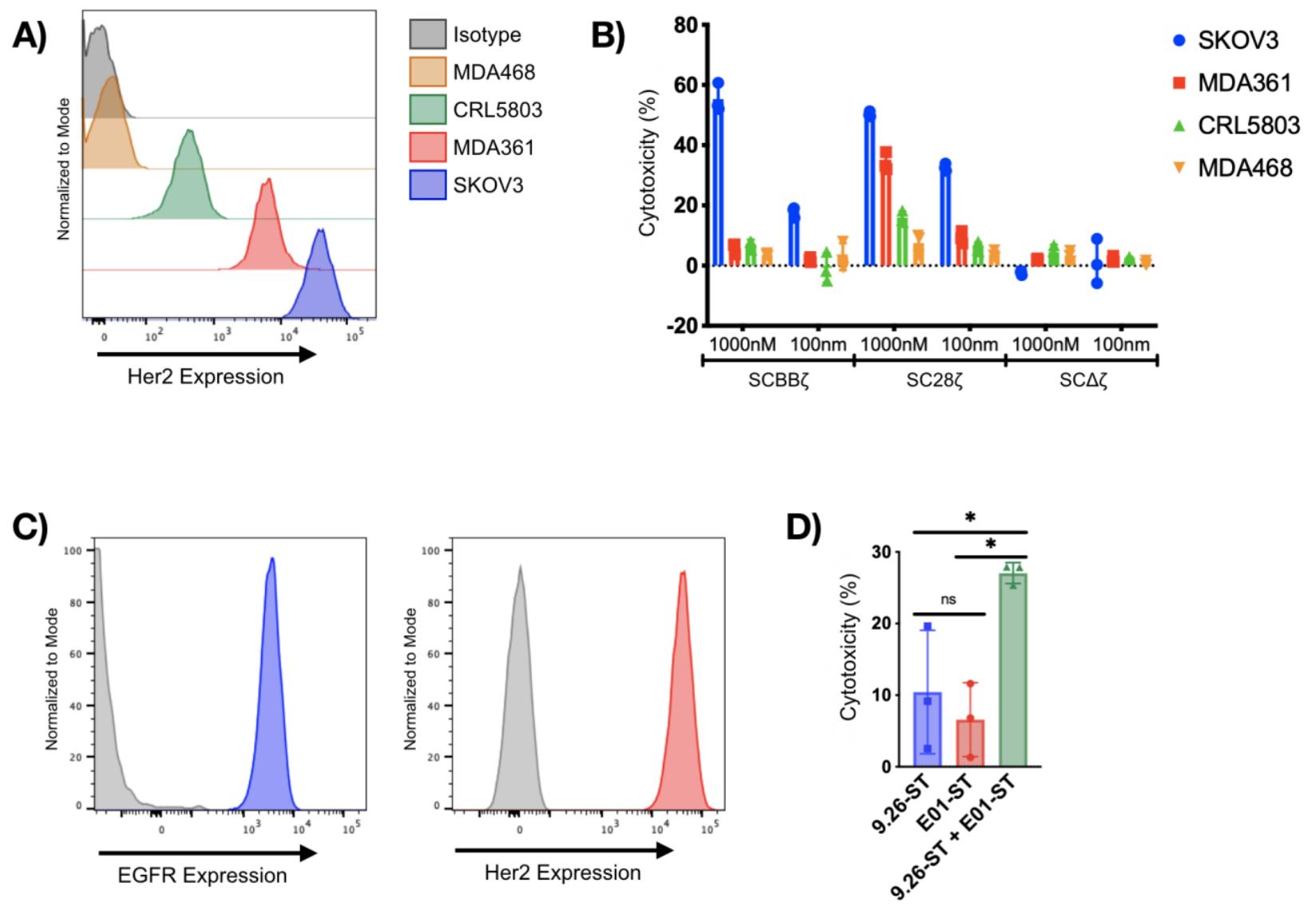

Supplemental Figure 3. Impact of antigen expression levels and dual arming on SpyCatcher T cell lytic function. (A) SKOV3, MDA-MB-361, CRL5803, and MDA-MB-468 cell lines stained for Her2 expression. (B) Lytic function of SpyCatcher T cells armed with 1000nM or 100nM Herceptin-ST against cells lines from (A). (C) SKOV3 cells stained for Her2 (red) and EGFR (blue) expression relative to isotype control (grey). (D) SpyCatcher-BBל T cells armed with either 50nM myc-9.26-ST ( $\alpha$ Her2; blue), 50nM Flag-Eo1-ST ( $\alpha$ EGFR; red), or both simultaneously at 50nM each (green) and co-cultured with SKOV3 tumor cells. Residual luciferase expression was calculated after 20 hours. All co-cultures were done using a 7:1 E:T ratio. Statistical significance for (D) was calculated using a one-way ANOVA with Tukey posttest. Error bars represent mean +/- standard deviation. Data points are averages of three replicates. Representative $\mathrm{T}$ cell donor is shown. $* \mathrm{P}<.05 ; * * \mathrm{P}<.01 ; * * * \mathrm{P}<.001$. 

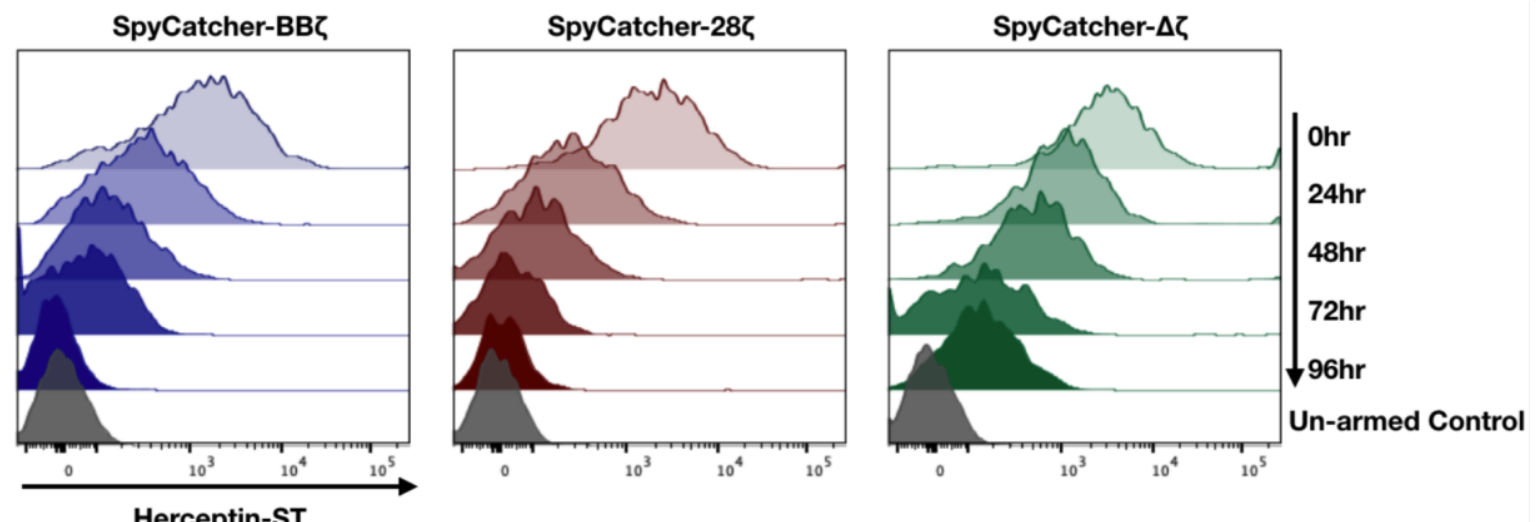

Supplemental Figure 4. Basal rate of armed SpyCatcher receptor turnover. Rested SpyCatcher T cells were maximally armed with Herceptin-ST and analyzed for loaded receptor every 24 hours to determine the rate of loaded receptor loss from the cell surface. Un-armed SpyCatcher $\mathrm{T}$ cells were stained as a negative control (grey histogram). 


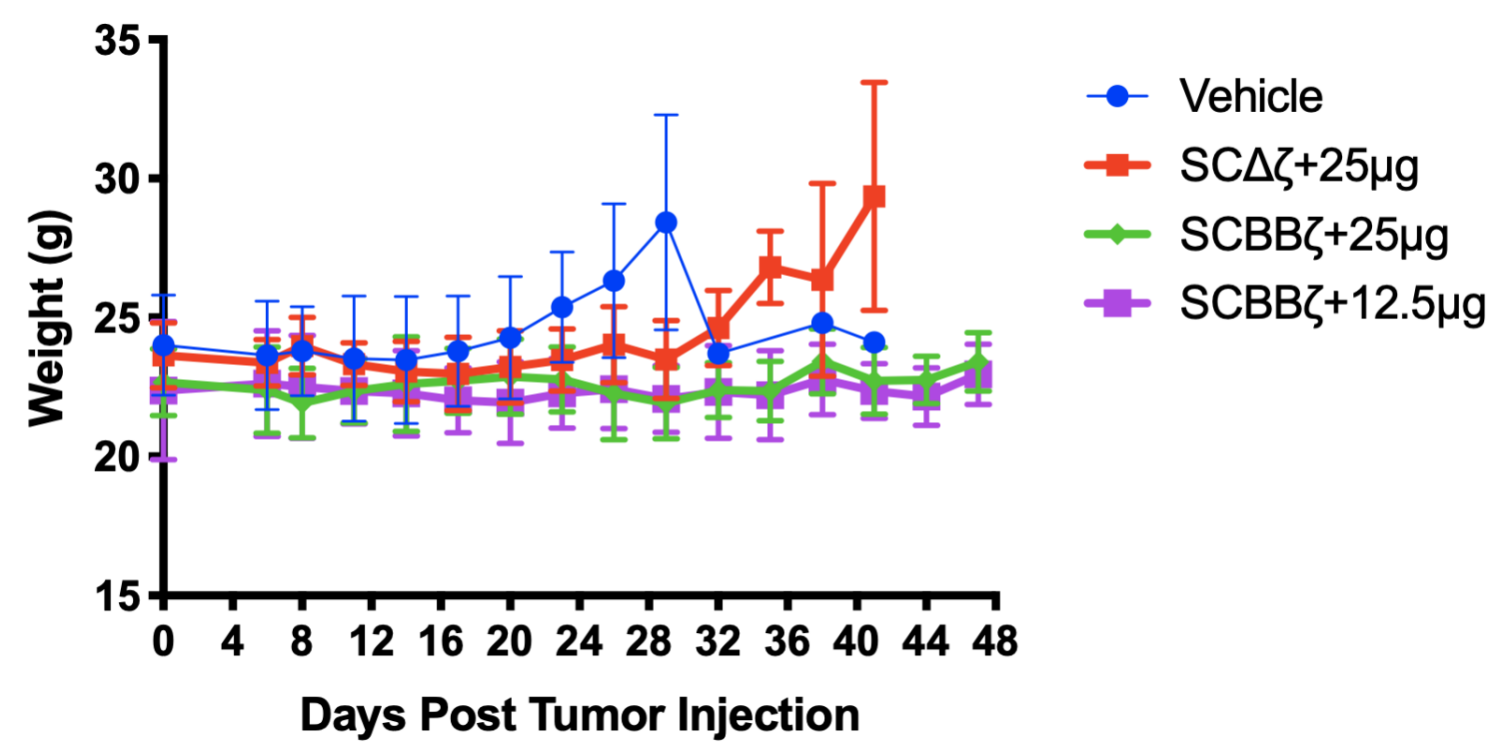

Supplemental Figure 5. Treatment with SpyCatcher-BB $\zeta$ T cells did not lead to changes in weight. NSG mice ( $\mathrm{n}=4$ per group) were injected intraperitoneally with $1 \times 10^{6}$ SKOV3 tumor cells on Day 0 , followed by $12.5 \times 10^{6}$ Herceptin-ST armed SpyCatcher-BB $\zeta \mathrm{T}$ cells on Day 7 . Herceptin-ST was administered on day 8, with subsequent injections every 3 days. Injection amounts indicate dose per mouse. Weight of each mouse was monitored during course of treatment. Weight change was not observed in treatment groups, while increases in weight change occurred in control groups due to ascites formation. Error bars represent mean $+/$ - standard deviation. 

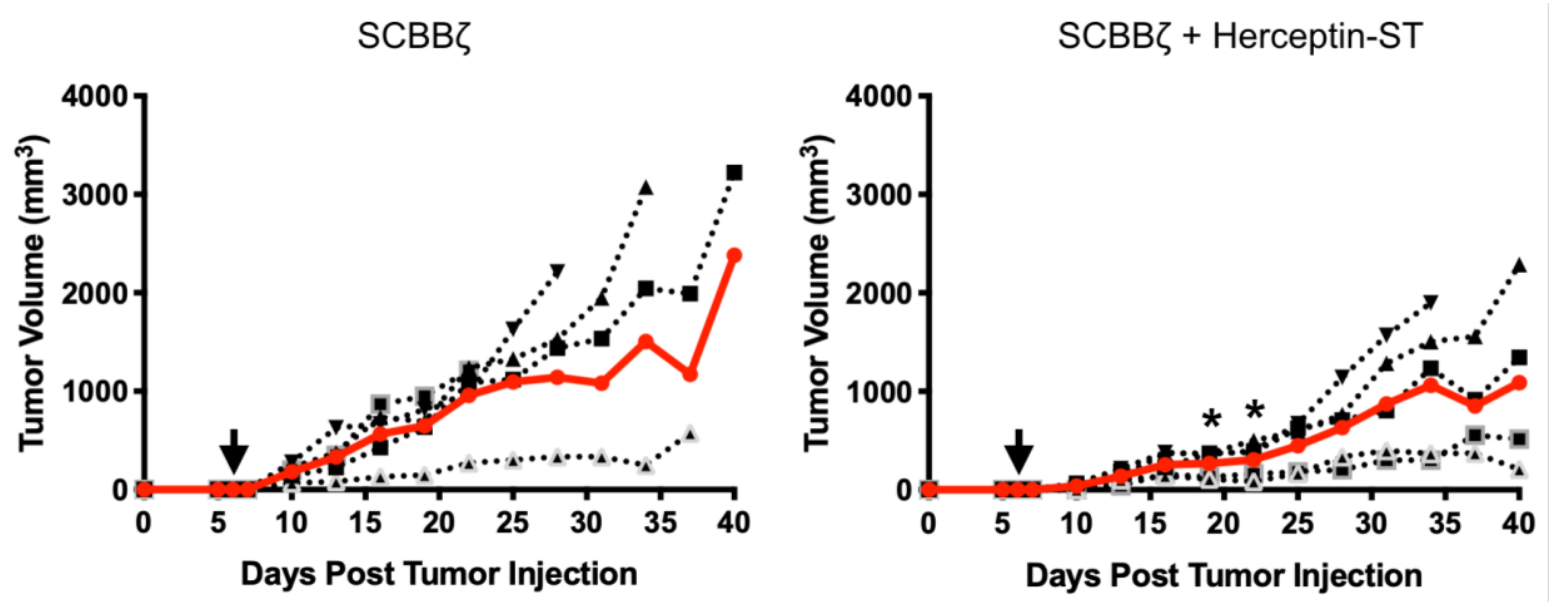

Supplemental Figure 6. SpyCatcher T cells slow the rate of tumor grown in aggressive S.C. tumor model. NSG mice ( $\mathrm{n}=5$ per group) were injected subcutaneously with $1 \times 10^{6}$ SKOV3 tumor cells on Day 0, followed by $12.5 \times 10^{6}$ SpyCatcher-BB $\zeta$ T cells armed with Herceptin-ST on Day 6. 50 $\mu$ g Herceptin-ST targeting ligand was administered one day after T cell infusion, followed by subsequent injections every 3 days for the entirety of the study. Tumor volume was monitored by caliper measurement. Black lines represent individual mice, while red lines represent the average tumor volume. Time points at which tumor growth was significantly reduced relative to SpyCatcher-BB $\zeta$ alone group are noted with an asterisk $(*)$. $* \mathrm{P}<.05 ; * * \mathrm{P}<.01 ; * * * \mathrm{P}<.001$. 
A)
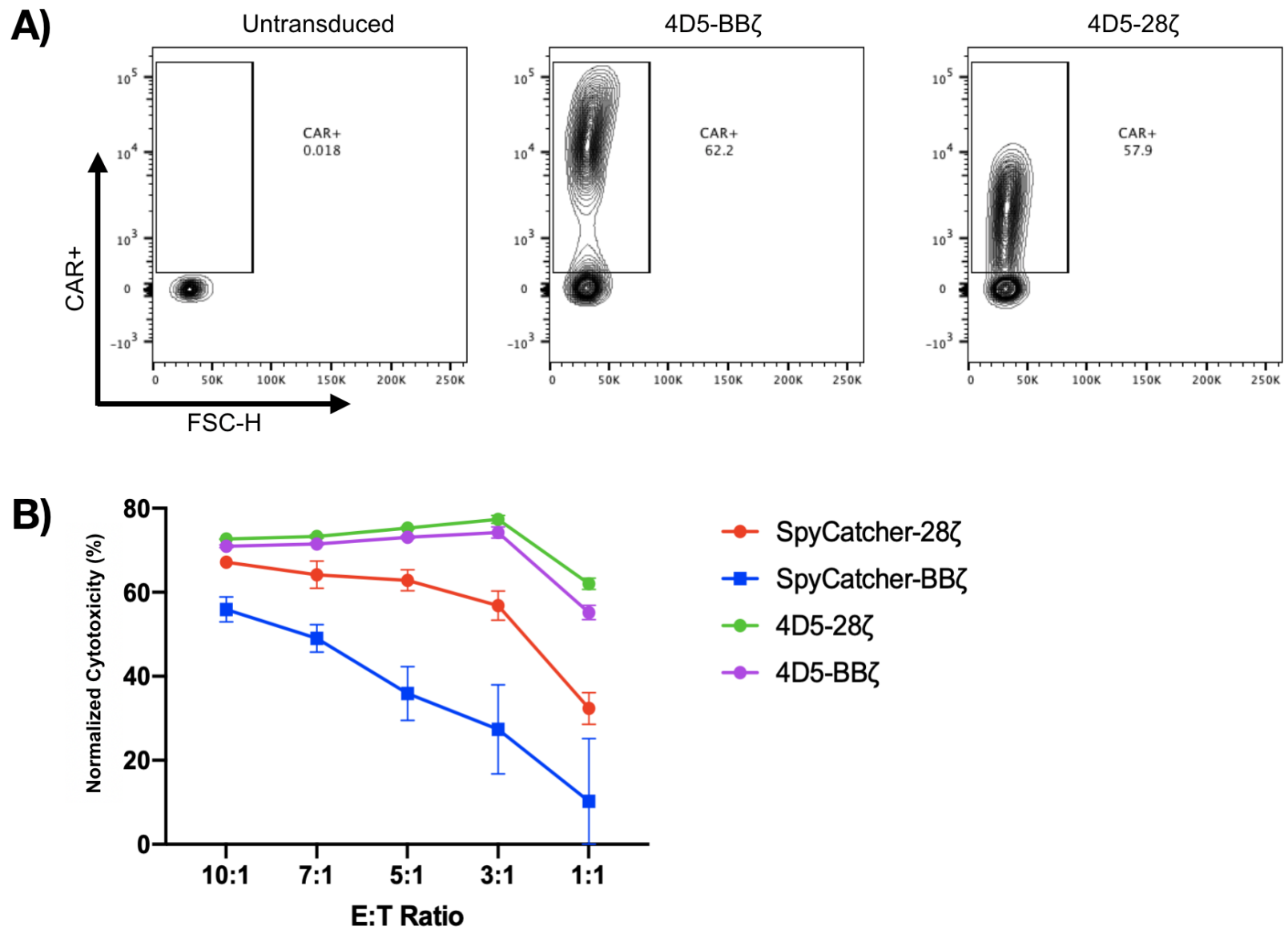

Supplemental Figure 7. Comparison of SpyCatcher $\mathbf{T}$ cell and CAR T cell lytic function. (A)

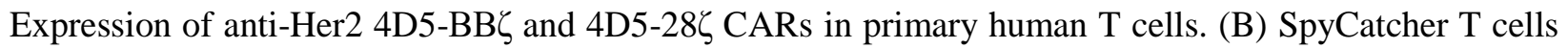
armed with 1000nM Herceptin-ST or 4D5 CAR T cells were co-cultured in the presence of SKOV3 (Her2+) tumor cells at various E:T ratios. Residual luciferase expression was calculated after 20 hours and normalized to untransduced $\mathrm{T}$ cell lysis at each E:T ratio using the following equation: [1-(effector wells)/(target cells alone) - 1-(untransduced T cells)/(target cells alone)]*100. Error bars represent mean +/- standard deviation. Data points are averages of three replicates. Representative T cell donor is shown. 
Supplemental videos. GFP-SpyCatcher-BB $\zeta$ T cells armed with 1000nM (V1), 100nM (V2), 10nM (V3), or OnM (V4) of Herceptin-ST demonstrate dose-dependent lysis of Her2+ SKOV3 cells expressing nuclear RFP. Tumor cell lysis was not observed by GFP-SpyCatcher- $\Delta \zeta \mathrm{T}$ cells armed with 1000nM (V5) Herceptin-ST. Addition of 100ng Herceptin-ST to unarmed GFP-SpyCatcher-BB $\zeta$ T cells co-cultured with Her2+ SKOV3 cells induces lytic function (V6; Herceptin-ST added at 48hrs). 